\title{
Modeling and Modal Analysis of Sliding Yaw Bearing in the Wind Turbine
}

\author{
Liu Yanping, \\ School of Energy Power and Mechanical Engineering \\ North China Electric Power University \\ Beijing China, 102206 \\ lyp@ncepu.edu.cn
}

\begin{abstract}
Sliding yaw bearing works in the complex environment with large load range, so its dynamic analysis is very important. The modal analysis of sliding yaw bearing is not only an effective method for analyzing sliding yaw bearing's dynamic characteristics, but also the basis of the dynamic respond analysis. This paper used the parameter design function of UG code to build the 3-D model of sliding yaw bearing and imported the model to ANSYS code to build finite element model. Then the finite element modal analysis was applied after meshing the model. Finally, the low-order natural frequency and the vibration mode were obtained. Therefore, the modal analysis of sliding yaw bearing can provide the basis of the dynamic characteristics analysis and optimization design of sliding yaw bearing. Meanwhile, the modal analysis can be considered as the reference to avoid yaw bearing resonance during wind turbine working.
\end{abstract}

Keywords- Wind turbine, sliding yaw bearing, UG code, modal analysis, ANSYS code

\section{INTRODUCTION}

Since sliding yaw bearing has simple structure, high load capacity, high reliability and low cost, more and more wind turbines use sliding yaw bearing. During the normal working process of wind turbine, there are not only static loads on the yaw bearing, but also varying dynamic loads. So, the sliding yaw bearing dynamic analysis is very important. Especially, when the period of the load variation is higher than the period of natural vibration, the dynamic respond has an obvious influence on the yaw bearing. So it's necessary to carry on the modal analysis of sliding yaw bearing to obtain the largest vibration mode and the relevant natural frequency, which can provide the reference for avoiding resonance during wind turbine working. In addition, the modal analysis of sliding yaw gearing is also the foundation of its dynamic characteristics analysis.

\section{Modal ANAlysis THEORY}

Modal analysis is mainly used to determine the vibration characteristics of components, namely the natural frequency and vibration modes, which are the major parameters of structures affected by dynamic loads. As sliding yaw bearing often works in complex conditions, it is necessary to carry on modal analysis of sliding yaw bearing to obtain the structure natural frequency and vibration type, and take indispensable measure during the design process to avoid the unnecessary loss caused by resonance.

\author{
Shang Lilong, Yu Yi \\ School of Energy Power and Mechanical Engineering \\ North China Electric Power University \\ Beijing China, 102206 \\ shang111988@126.com
}

The motion differential equation of multi-degree of freedom is shown as follows [1]:

$[M]\{\ddot{X}\}+[C]\{\dot{X}\}+[K]\{X\}=\{F(t)\}$

where $[\mathrm{M}]$,

$[\mathrm{C}]$, and $[\mathrm{K}]$ represent the system mass, damping and stiffness matrices, respectively.

$\{\ddot{X}\},\{\dot{X}\}$, and $\{\mathrm{X}\}$ donate acceleration, velocity and displacement vectors, respectively. $\{\mathrm{F}(\mathrm{t})\}$ represents the vibration vector.

The purpose of the modal analysis for sliding yaw bearing is to obtain the natural frequency and vibration modes, which are independent of the external load of the system. So the structure damping has little influence on natural frequency and can be ignored, and we can set $\{\mathrm{F}(\mathrm{t})\}=0$ during the modal analysis process. Taking into account all above conditions, the motion equation of free vibration without damping is given by:

$$
[M]\{\ddot{X}\}+[K]\{X\}=0
$$

Its corresponding characteristic equation can be written as:

$$
\left([K]-\omega^{2}[M]\right)\{X\}=\{0\}
$$

where $\omega$ is the natural frequency of the system.

Eq. (3) is the $\mathrm{N}$ times equation of $\omega$, while $\mathrm{N}$ is the number of the node DOF. Nth natural frequency can be obtained by solving Eq. (3) [2]. For engineering structures, only the lower orders frequency should be considered. Here, each $\omega$ corresponds to an $\{X\}$, which is the corresponding main vibration mode.

\section{Parametric Modeling Of Sliding Yaw Bearing}

The basis of parametric modeling is that the model changes automatically when the defined parameters change [3]. Currently, the common parametric modeling codes include PRO/E, UG, SolidWorks, solidEdge, etc. Comparing with other parametric modeling codes, UG code has better interface with ANSYS code. So it is easier to import the model programmed by UG code into ANSYS code. On the other hand, the curve surface of sliding yaw bearing is complex, and the tools of curve surface analysis and formation in UG code are very plenty [4]. Therefore, our research adopts UG code for modeling of the sliding yaw bearing. 
The example in this paper is the model of sliding yaw bearing applied on the $1.5 \mathrm{MW}$ wind turbine of certain company. The model is a large scale bearing with gear tooth.

\section{A. MODELING OF YAW GEAR RING}

The contour of yaw gear ring is firstly established and rotates full 360 degrees on the $\mathrm{Z}$ axis to obtain yaw gear ring model. Secondly, gear ring parameters and relevant data are imported to UG code. Finally, according to the involute parameters and the principles of involute, the gear tooth profile is completed by the mirror function of UG code and the necessary curve cutting and stretching on the entity. The gear tooth circular array is achieved through the entity array toolbar. The parametric design of yaw gear ring is then finished, and the 3-D model of yaw gear ring is accurately established.

\section{B. MODELING OF SIDE BEARING}

First, the sketch of side bearing can be obtained through the fast clipping tools. Then the sketch is stretched respectively to form the first side bearing model by summation operator with boolean operation. The last step is to shear model appearance and add blots, nuts and other accessories.

\section{YAW BEARING ASSEMBLING}

With the entity array toolbar and the number of side bearings, eight, side bearings are set as polar arrayed and Z axis is the reference axis. Then, side bearings are assembled to the yaw gear ring which is the basis, and the origin is the reference point. The model of sliding yaw bearing with gear ring is shown in Fig.1.

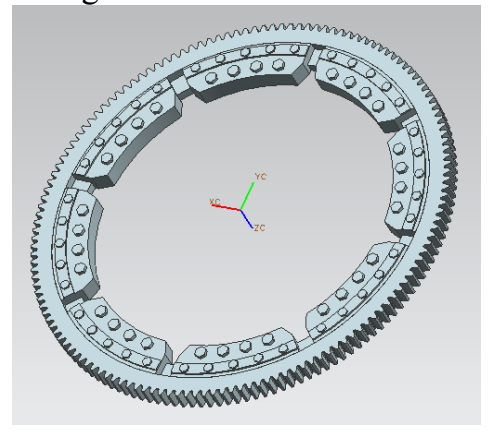

Figure 1. Cutting diagram of yaw bearing

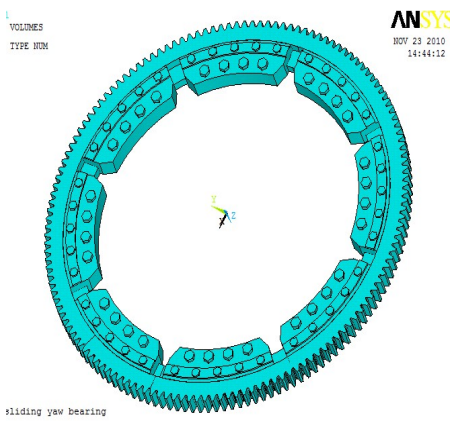

Figure 2. Finite element model of yaw bearing

\section{Modal Analysis Of Sliding Yaw Bearing}

ANSYS code provides seven methods for extracting modal, i.e. Subspace method, Block Lanzcos method, Power Dynamics method, Reduce method, Unsymmetric method, Damped method and QR Damped method. In our research, Block Lanzcos method is appropriate for calculating natural frequency of the system whose eigenvalue spectrum has a certain range. Through this method, the rate of convergence for solving the natural frequency from neutral frequency to high frequency is almost the same as that from neutral frequency to low frequency in frequency spectrum. Moreover, Block Lanzcos method has higher accuracy and is particularly applicable to solve the problem of the eigenvalue of large scale symmetrical structure [5]. So this research applies Block Lanzcos method for the modal analysis of sliding yaw bearing.

\section{A. BUILD THE FINITE ELEMENT MODEL}

Firstly, the 3-D model of sliding yaw bearing established by UG code is imported to ANSYS code and the finite element model is obtained, as shown in Fig. 2.

ANSYS code can carry on modal analysis of pre-stressed and circularly symmetric structures. The latter modal analysis of structure completes the overall structure through the modal analysis of a part of the circularly symmetric structure [5]. The model used in this research is a circularly symmetric structure. Only $1 / 8$ of the overall yaw bearing is analyzed, so that the analysis procedure occupies less computer memory and runs faster. After built in UG code, the modal analysis is imported to ANSYS code, as shown in Fig.3.

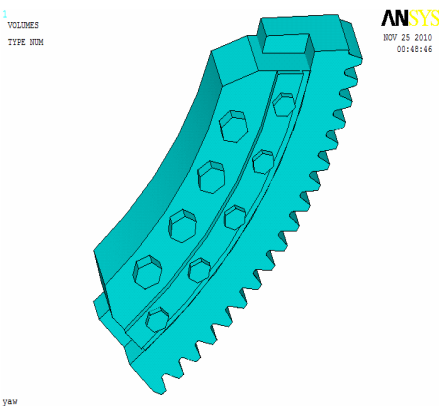

Figure 3. Finite element model of yaw bearing

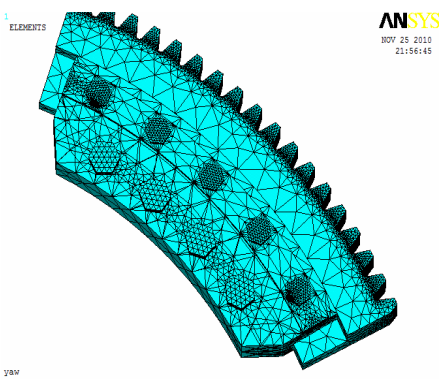

Figure 4. Meshing the model of yaw bearing 


\section{B. DEFINE BASIC PARAMETERS AND MESH}

Element type, elastic modulus, density and other parameters are defined in this section. This research uses the element type of solid 186 to mesh and the elastic modulus is set as $2.1 * 1011 \mathrm{~Pa}$ where the Poisson ratio is set as 0.3 and density is set as $7800 \mathrm{~kg} / \mathrm{m} 3$. The model after meshing is shown in Fig.4.

\section{APPLY LOAD, SOLVE AND POSTPROCESS}

In practice, the gear ring of the sliding yaw bearing is attached to the flange on the top of wind turbine tower. Meanwhile, side bearings are connected to the retainer of nacelle by high strength bolts. Both the gearing ring and side bearings are constrained with the displacement and rotation which is called all degree-of-freedom constraints. According to the design manual, when the vibration of bearing is analyzed, the frequency range is from $50 \mathrm{~Hz}$ to $10000 \mathrm{~Hz}$. Thus, the initial frequency and the stop frequency of modal analysis are equal to $50 \mathrm{~Hz}$ and $10000 \mathrm{~Hz}$, respectively. After applying boundary conditions, ten natural frequency of modal and displacement are shown in Tab. 1. The vibration modes are shown in Fig. 5.

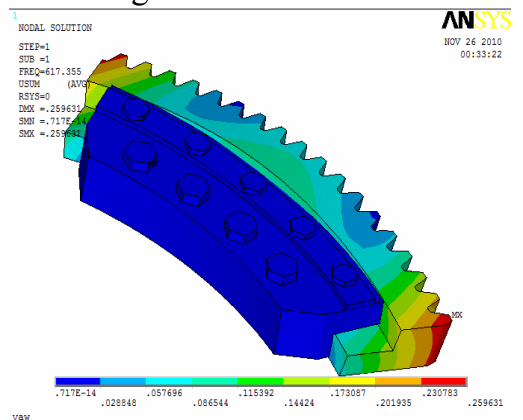

a) The first vibration mode diagram

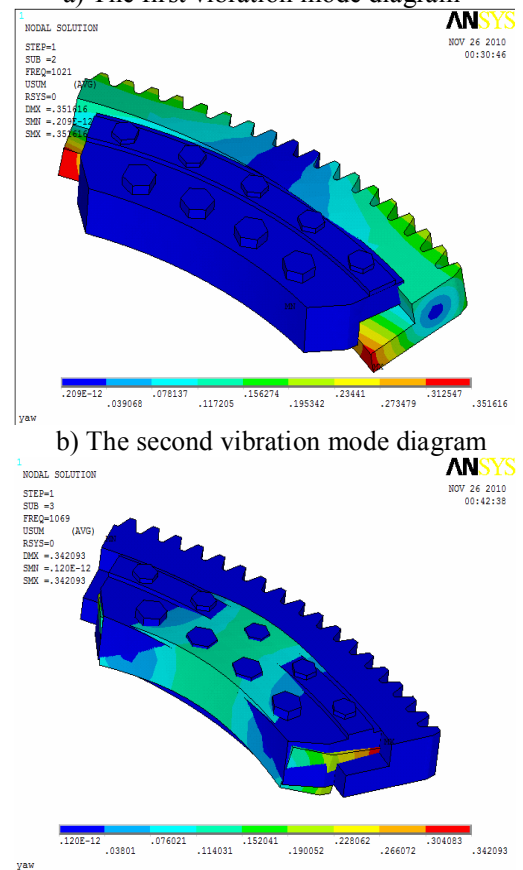

c) The third vibration mode diagram

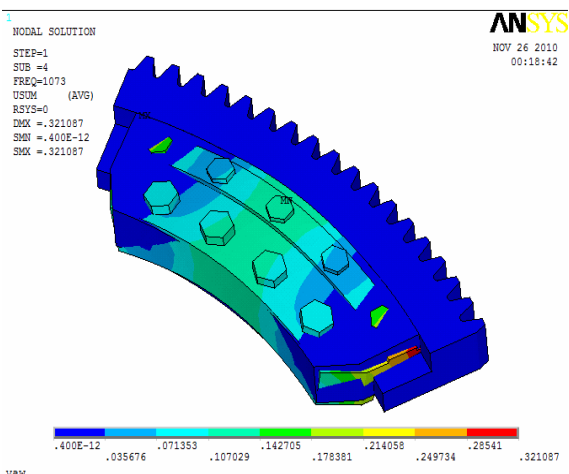

d) The fourth vibration mode diagram

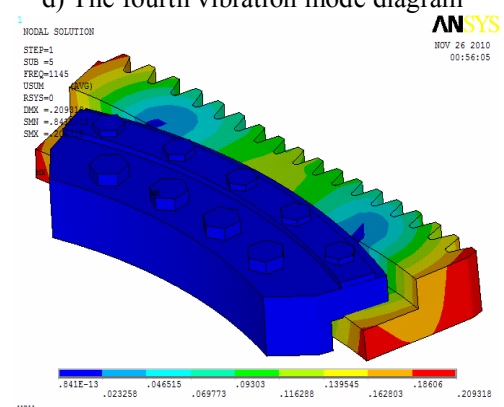

e) The fifth vibration mode diagram
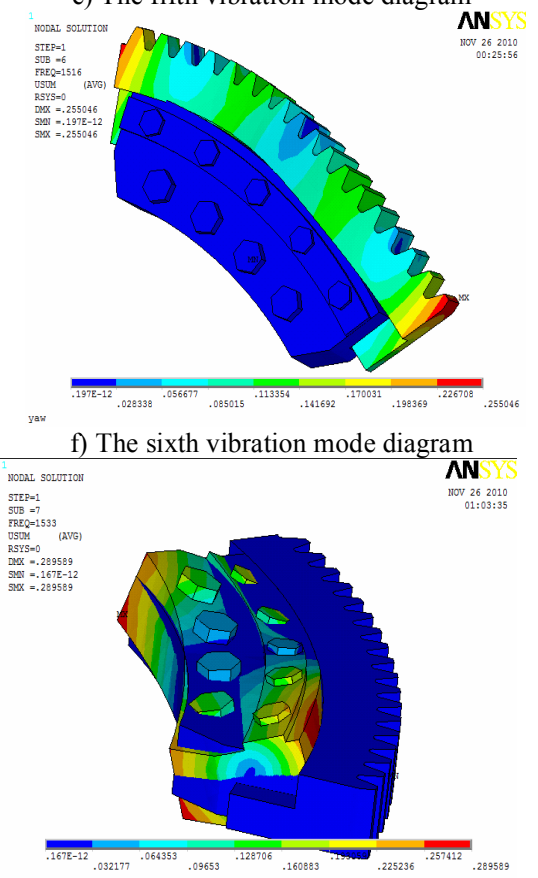

g) The seventh vibration mode diagram 


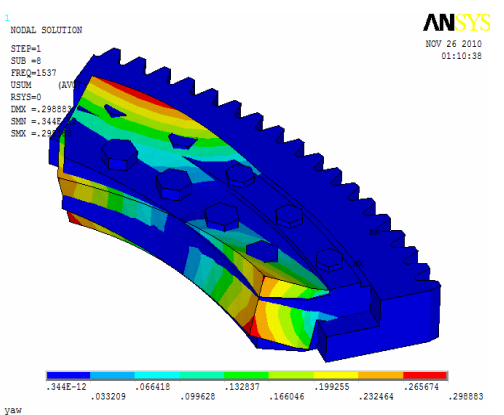

h) The eighth vibration mode diagram

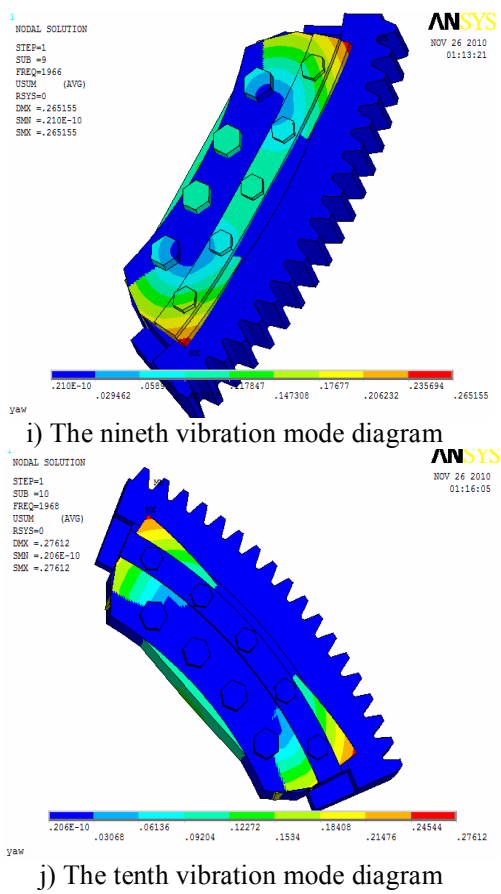

Figure 5. Ten-order vibration model diagram

\section{CONCLusions}

(1) The curve surface of sliding yaw bearing is complex. UG code can be used to establish the model of sliding yaw bearing by its parametric modeling functions and plentiful curve surface analysis functions. So, the model is easier to be imported to ANSYS code for analysis.

(2) According to the results of modal analysis, the maximum vibration mode appears when the natural frequency is $1021.4 \mathrm{~Hz}$. So, the frequency of dynamic system should be far away from this natural frequency, $1021.4 \mathrm{~Hz}$, to avoid yaw bearing resonance.

(3) In the mode diagrams, the displacement of side bearings changes obviously when the maximum vibration mode appears. In the other words, the marrying up condition between side bearings and yaw gear ring is the main factor that affects the overall yaw bearing system.

(4) Vibration modes are the relative values of amplitude between nodes. They can be used to determine the vibration form and weak position of the system. According to the vibration mode diagrams of the bearing finite element model, it's obvious that the pre-stressed structure is necessary for the vibration reduction.

Therefore, the mass and stiffness distribution affect the natural frequency and vibration modes of the sliding yaw bearing. The optimized natural frequency can be obtained through the adjustment of the mass and stiffness in the sliding yaw bearing. The results of this research provide the basis for yaw bearing optimization design. Meanwhile, this research is the preliminary work of dynamic design for the sliding yaw bearing.

\section{REFERENCES}

[1] Zhang, Nan. Model analysis of tower crane drive gear based on ANSYS. Construction Mechanization [J], 2010.3, PP: 53-55.

[2] Xia, Min-min; Li, Li-jun; Zhan, Chun-sheng. Modal analysis on liquid hydrostatic bearing of gear shaping machine based on ANSYS. Mechanical Research \& Application [J], 2010.1, PP: 94-95.

[3] Cui, Liang. Parameter design and FEM analysis of cylinder gear based on UG. Engineer [J], 2010.3, PP: 111-113.

[4] Wang, Ting-ting; Zhang, Hong; Zeng, Zi-jian. Study of meshing CAD models methods in ANSYS. Tractor \& Farm Transporter [J], 2008.2, PP: 41-42, 45.

[5] Liu, Xiang-xin; Meng, Xian-yi; Guo, Xu-wei. Ansys basic and applied tutorial. Science Press[M], 2006, PP: 12-134.

TABLE I. THE FIRST 10 NATURAL FREQUENCIES OF THE YAW BEARING FINITE ELEMENT MODEL

\begin{tabular}{|c|c|c|c|c|c|}
\hline Order & $\begin{array}{c}\text { Natural } \\
\text { frequency(Hz) }\end{array}$ & $\begin{array}{c}\text { Maximum } \\
\text { vibration } \\
\text { modal(m) }\end{array}$ & Order & $\begin{array}{c}\text { Natural } \\
\text { frequency(Hz) }\end{array}$ & $\begin{array}{c}\text { Maximum } \\
\text { vibration } \\
\text { modal(m) }\end{array}$ \\
\hline one & 617.35 & 0.259631 & six & 1515.6 & 0.255046 \\
\hline two & 1021.4 & 0.351616 & seven & 1533.2 & 0.289589 \\
\hline three & 1069.2 & 0.342093 & eight & 1537.0 & 0.298883 \\
\hline four & 1072.6 & 0.321087 & nine & 1965.9 & 0.265155 \\
\hline five & 1144.6 & 0.209318 & ten & 1968.5 & 0.27612 \\
\hline
\end{tabular}

